DFF $1 / 04 / 04$

\title{
Projectors, matrix models and noncommutative monopoles
}

\author{
P. Valtancoli \\ Dipartimento di Fisica, Polo Scientifico Universitá di Firenze \\ and INFN, Sezione di Firenze (Italy) \\ Via G. Sansone 1, 50019 Sesto Fiorentino, Italy
}

\begin{abstract}
We study the interconnection between the finite projective modules for a fuzzy sphere, determined in a previous paper, and the matrix model approach, making clear the physical meaning of noncommutative topological configurations.
\end{abstract}




\section{Introduction}

One of the more interesting applications of noncommutative geometry is the study of topologically nontrivial configurations over a noncommutative manifold. In the commutative case, a general method, based on the Serre-Swan's theorem allows to paraphrase the study of nontrivial topologies for vector bundles in terms of projective modules. At a classical level this theorem asserts the complete equivalence between the category of vector bundles over a compact manifold $M$ and the category of finite projective modules over the commutative algebra $C(M)$ of ( smooth) functions over $M$.

The aim of this paper is to make an analogous bridge at a noncommutative level, by studying an explicit example, i.e. noncommutative monopoles on a fuzzy sphere [1]. In Ref. [2] ( see also [3]-[5] ) we identified the noncommutative projectors which should clarify the nontrivial topologies on a fuzzy sphere, however to apply them in a physical framework we need to deconstruct the projectors and to make a bridge with the more familiar language of connections [6]-[7]. On the other hand in Refs. [8]-[9] we studied a matrix model, firstly introduced in refs. [10]-13] which defines $U(1)$ noncommutative gauge theory on a fuzzy sphere, based on a matrix variable $X_{i}$ [14]-[20, which contains the information about the connection. We then investigated in Ref. [9] the possible soliton solutions, but we didn't succeed to identify the nontrivial topologies at the connection level.

Therefore our aim is to complete our knowledge on noncommutative gauge theories on a fuzzy sphere by reaching mainly two objectives: firstly we are able to identify the class

of models for which the projectors of Ref. [2] are indeed solution of the $Y-M$ equations of motion, and secondly after deconstructing the projectors in terms of more fundamental vector-valued fields, we are able to identify the nontrivial connections.

The structure of the paper is as follows. Firstly we recall the construction ( see [21] ) of projectors for a classical monopole on $S^{2}$ by using the Hopf principal fibration $\pi: S^{3} \rightarrow S^{2}$ on the two-sphere, with $U(1)$ as a structure group. Then we write the $Y-M$ equations of motion in terms of a classical matrix model and show that the classical projectors are solution to them. In section 4 we recall the construction of noncommutative projectors on a fuzzy sphere and, using a simple relation between the matrix model variable $X_{i}$ and the noncommutative projectors we identify the class of models which allow for nontrivial topologies as solutions to the $Y-M$ equations of motion. Finally we reconstruct the gauge connection from the projectors and we give a nice interpretation of a topologically nontrivial matrix variable $X_{i}$. 


\section{Monopole solution in terms of projectors}

In Ref. 21] it has been shown how to characterize the nontrivial connections on the twosphere $S^{2}$ in terms of projectors ( gauge invariant definition of a connection ), by using the Hopf principal fibration $\pi: S^{3} \rightarrow S^{2}$ on the two-sphere $S^{2}$, with $U(1)$ as structure group.

The general procedure is starting from the algebra of $N \times N$ matrices whose entries are elements of the smooth function algebra $C^{\infty}\left(S^{2}\right)$ on the base space $S^{2}$, i.e. $M_{N}\left(C^{\infty}\left(S^{2}\right)\right)$. The section module of the bundle on which the monopole lives can be identified with the action of a global projector $p \in M_{N}\left(C^{\infty}\left(S^{2}\right)\right)$ on the trivial module $\left(C^{\infty}\left(S^{2}\right)\right)^{N}$, i.e. the right module $p\left(C^{\infty}\left(S^{2}\right)\right)^{N}$, where un element of $\left(C^{\infty}\left(S^{2}\right)\right)^{N}$ is the vector

$$
\| f>>=\left(\begin{array}{c}
f_{1} \\
\cdots \\
f_{N}
\end{array}\right)
$$

with $f_{1}, f_{2}, \ldots, f_{N}$ elements of $\left(C^{\infty}\left(S^{2}\right)\right)^{N}$.

The projectors are written in general as ket-bra valued functions ( with $N \times N$ entries )

$$
p=|\psi><\psi| \quad<\psi\left|=<\psi_{1}, \ldots, \psi_{N}\right| .
$$

The normalization condition on the vector valued function

$$
<\psi \mid \psi>=1
$$

automatically implies that $p$ is a projector since

$$
p^{2}=|\psi><\psi| \psi><\psi \mid=p \quad p^{\dagger}=p
$$

and that the projector is of rank 1 over $C$ since

$$
\operatorname{Tr}(p)=<\psi \mid \psi>=1
$$

We notice that it is possible to redefine the vector valued function $|\psi\rangle$ up to the right action of an element $w \in U(1)$, leaving the projector $p$ invariant.

The associated canonical connection is defined as : 


$$
\nabla=p \cdot d
$$

and its curvature

$$
\nabla^{2}=p(d p)^{2}=|\psi><d \psi| d \psi><\psi \mid .
$$

The corresponding 1-form connection $A_{\nabla}$ has a very simple expression in terms of the vector-valued function $|\psi\rangle$

$$
A_{\nabla}=<\psi \mid d \psi>.
$$

The classification of gauge non-equivalent connections depends on the possible global left actions on the vector valued function $|\psi\rangle$. If this global left action is reduced to the unitary group $S U(N)=\left\{s \mid s s^{\dagger}=1\right\}$ preserving the normalization

$$
\left.|\psi>\rightarrow| \psi^{s}\right\rangle=s\left|\psi>\quad<\psi^{s}\right| \psi^{s}>=1
$$

then we remain in the same class of solutions since the connection $A_{\nabla}$ is left invariant

$$
A_{\nabla}^{s}=<\psi^{s}\left|d \psi^{s}>=\psi\right| s^{\dagger} s d \psi>=A_{\nabla} .
$$

To obtain gauge non equivalent connections we should act with group elements which do not preserve the normalization.

The explicit construction of nontrivial connections on the sphere depicted in ref [21] involves the Hopf fibration. The $U(1)$ principal fibration $\pi: S^{3} \rightarrow S^{2}$ is explicitly realized as follows. The initial space is the 3-dimensional sphere:

$$
S^{3}=\left\{\left(z_{0}, z_{1}\right) \in C^{2} ;\left|z_{0}\right|^{2}+\left|z_{1}\right|^{2}=1\right\}
$$

with right $U(1)$-action

$$
S^{3} \times U(1) \rightarrow S^{3}, \quad\left(z_{0}, z_{1}\right) \cdot w=\left(z_{0} w, z_{1} w\right) .
$$

The bundle projection $\pi: S^{3} \rightarrow S^{2}$ defining the Hopf fibration $\pi\left(z_{0}, z_{1}\right)=\left(x_{1}, x_{2}, x_{3}\right)$ is determined to be 


$$
\begin{aligned}
& x_{1}=z_{0} \bar{z}_{1}+z_{1} \bar{z}_{0} \\
& x_{2}=i\left(z_{0} \bar{z}_{1}-z_{1} \bar{z}_{0}\right) \\
& x_{3}=\left|z_{0}\right|^{2}-\left|z_{1}\right|^{2} .
\end{aligned}
$$

We recall the projector construction for monopoles of charge $-n, n \in N$. Let us consider the following vector-valued function with $N=n+1$ components

$$
<\psi_{-n} \mid=\left(z_{0}^{n}, \ldots, \sqrt{\left(\begin{array}{c}
n \\
k
\end{array}\right)} z_{0}^{n-k} z_{1}^{k}, \ldots, z_{1}^{n}\right)
$$

where, for the general $n$-case we need to introduce the binomial coefficients

$$
\left(\begin{array}{l}
n \\
k
\end{array}\right)=\frac{n !}{k !(n-k) !} \quad k=0,1, . . n .
$$

Since the coordinates $\left(z_{0}, z_{1}\right)$ belong to the sphere $S^{3}$, the normalization condition for the vector-valued function (2.14) is satisfied by construction :

$$
<\psi_{-n} \mid \psi_{-n}>=\left(\left|z_{0}\right|^{2}+\left|z_{1}\right|^{2}\right)^{n}=1
$$

From $<\psi_{-n} \mid$ we can deduce the projector

$$
p_{-n}=\left|\psi_{-n}><\psi_{-n}\right|
$$

The normalization condition (2.16) ensures that $p_{-n}$ is a projector

$$
\begin{aligned}
p_{-n}^{2} & =\left|\psi_{-n}><\psi_{-n}\right| \psi_{-n}><\psi_{-n}|=| \psi_{-n}><\psi_{-n} \mid=p_{-n} \\
p_{-n}^{\dagger} & =p_{-n}
\end{aligned}
$$

and of rank 1 since its trace is the constant 1 :

$$
\operatorname{Tr} p_{-n}=<\psi_{-n} \mid \psi_{-n}>=1
$$

While $<\psi_{-n} \mid$ is defined on the sphere $S^{3}$, the projector $p_{-n}$ is defined on the sphere $S^{2}$, since the left $U(1)$-action of $<\psi_{-n} \mid$ leaves the projector $p_{-n}$ invariant: 


$$
\begin{aligned}
<\psi_{-n} \mid & \rightarrow<\psi_{-n}^{w}\left|=w^{n}<\psi_{-n}\right| \quad \forall w \in U(1) \\
p_{-n} & \rightarrow p_{-n}^{w}=p_{-n} \quad \bar{w} w=1 .
\end{aligned}
$$

Therefore the projector entries can be expressed entirely as functions of the base space $S^{2}$, as it should be.

To generalize the projector construction for monopoles of charge $n$, we consider the vector-valued function:

$$
<\psi_{n} \mid=\left(\bar{z}_{0}^{n}, \ldots, \sqrt{\left(\begin{array}{c}
n \\
k
\end{array}\right)} \bar{z}_{0}^{n-k} \bar{z}_{1}^{k}, \ldots, \bar{z}_{1}^{n}\right)
$$

that is again normalized to 1 .

The corresponding projector

$$
p_{n}=\left|\psi_{n}><\psi_{n}\right|
$$

is invariant under the $U(1)$ action

$$
<\psi_{n}\left|\rightarrow<\psi_{n}^{w}\right|=\bar{w}^{n}<\psi_{n} \mid \quad \forall w \in U(1) .
$$

In this classical case since the functions $<\psi_{-n} \mid$ and $<\psi_{n} \mid$ are related by complex conjugation, the corresponding projectors are related by hermiticity:

$$
p_{n}=\left(p_{-n}\right)^{\dagger}
$$

The corresponding monopoles connections are proportional to the charge number $n$ :

$$
\begin{aligned}
A_{\mp n} & =<\psi_{\mp n} \mid d \psi_{\mp n}>=\mp n\left(\bar{z}_{0} d z_{0}+\bar{z}_{1} d z_{1}\right)=\mp n A_{1} \\
A_{1} & =\bar{z}_{0} d z_{0}+\bar{z}_{1} d z_{1} .
\end{aligned}
$$

The integer $n$ is related to the Chern number

$$
c_{1}\left(p_{\mp n}\right)=-\frac{1}{2 \pi i} \int_{S^{2}} \operatorname{Tr}\left(\nabla_{\mp n}^{2}\right)=-\frac{1}{2 \pi i} \int_{S^{2}} \operatorname{Tr}\left(p_{\mp n}\left(d p_{\mp n}\right)^{2}\right)= \pm n .
$$




\section{Classical Yang-Mills action and matrix models}

The monopole connection is not only a topological property of $U(1)$ Yang-Mills theory on the two-sphere $S^{2}$ but it also satisfies the equations of motion. Therefore our aim is rewriting the Yang-Mills equations of motion in a form which makes manifest that the projectors of the classical monopoles are solution to them. This form will be used to make the extension to the noncommutative case.

Based on our experience on the possible representations of the Yang-Mills action, the most convenient choice turns out to rewrite the classical Yang-Mills action in terms of matrix models and then to connect the matrix model variables with the projectors.

Let us recall the most general $U(1)$ Yang-Mills action on the sphere in terms of matrix models [10]-13]. Let us define the matrix variable

$$
X_{i}^{0}=L_{i}+A_{i} \quad\left[L_{i}, .\right]=-i k_{i}^{a} \partial_{a}
$$

where $A_{i}$ is related to the Yang-Mills connection $A_{a}(\Omega)$ and to an auxiliary scalar field $\phi(\omega)$ as follows:

$$
A_{i}(\Omega)=k_{i}^{a} A_{a}(\Omega)+\frac{x_{i}}{R} \phi(\Omega) \quad \Omega=(\theta, \phi)
$$

and $k_{i}^{a}$ are the Killing vectors on the sphere $S^{2}$, and $R$ is its radius.

The classical action is defined as :

$$
\begin{aligned}
S(\lambda) & =S_{0}+\lambda S_{1}=-\frac{1}{g_{Y M}^{2}} \int d \Omega\left[\frac{1}{4}\left[X_{i}^{0}, X_{j}^{0}\right]\left[X_{i}^{0}, X_{j}^{0}\right]-\frac{2}{3} i \lambda \epsilon^{i j k} X_{i}^{0} X_{j}^{0} X_{k}^{0}\right. \\
& \left.+(1-\lambda) X_{i}^{0} X_{i}^{0}\right] .
\end{aligned}
$$

Defining a gauge covariant field strength

$$
\begin{aligned}
F_{i j} & =\left[X_{i}^{0}, X_{j}^{0}\right]-i \epsilon^{i j k} X_{k}^{0}= \\
& =\left[L_{i}, A_{j}\right]-\left[L_{j}, A_{i}\right]-i \epsilon_{i j k} A_{k}
\end{aligned}
$$

it can be developed in terms of the components fields (3.2) as follows:

$$
F_{i j}(\Omega)=k_{i}^{a} k_{j}^{b} F_{a b}+\frac{i}{R} \epsilon_{i j k} x_{k} \phi-i \frac{x_{j}}{R} k_{i}^{a} \partial_{a} \phi+i \frac{x_{i}}{R} k_{j}^{a} \partial_{a} \phi
$$


where $F_{a b}=-i\left(\partial_{a} A_{b}-\partial_{b} A_{a}\right)$ for the $U(1)$ case.

The classical Yang-Mills action on the sphere is thus reconstructed:

$$
\begin{aligned}
S(\lambda) & =-\frac{1}{4 g_{Y M}^{2}} \int d \Omega\left[\left(F_{a b}+(4-2 \lambda) i \epsilon_{a b} \phi \sqrt{g}\right)\left(F^{a b}+(4-2 \lambda) i \epsilon^{a b} \frac{\phi}{\sqrt{g}}\right)+\right. \\
& \left.-2 \partial_{a} \phi \partial^{a} \phi+\left(8(2-\lambda)^{2}-4(2-\lambda)\right) \phi^{2}\right] .
\end{aligned}
$$

The auxiliary scalar field $\phi$ can be decoupled from pure Yang-Mills theory for the particular value $\lambda=2$, in which case the action reduces to :

$$
\begin{aligned}
S(2) & =-\frac{1}{4 g_{Y M}^{2}} \int d \Omega\left(F_{a b} F^{a b}-2 \partial_{a} \phi \partial^{a} \phi\right) \\
d \Omega & =\sqrt{g} d \theta d \phi=\sin \theta d \theta d \phi \quad F^{a b}=g^{a a^{\prime}} g^{b b^{\prime}} F_{a^{\prime} b^{\prime}} .
\end{aligned}
$$

The equations of motion of the decoupled action $S(2)$ has two basic solutions:

i) the monopole solution, with $\phi=0$, which is the aim of the present paper;

ii) $A_{a}=0, \phi=$ const, which has been considered in the appendix of our paper [9]. In the following we will clarify that a simple noncommutative map is able to connect the noncommutative generalization of these two types of classical solutions.

We notice that rewriting the classical action in terms of matrix models, that becomes polynomial in the fundamental variables, making simpler the link with the method of projectors.

It is easy to observe that the projector $p_{0}$ can be linked to the matrix variables $X_{i}^{0}$ according to the following formula:

$$
\tilde{X}_{i}=p_{0} L_{i} p_{0}
$$

Being the projector a gauge invariant formulation of the connections, the matrix variable $\tilde{X}_{i}$ is again gauge invariant and therefore it is not directly connected with $X_{i}^{0}$. In any case shifting to the gauge covariant formulation is still possible, since the projector $p_{0}$ can be put in the form of a ket-bra valued function:

$$
p_{0}=|\psi><\psi|
$$

and, once the vector-valued function $<\psi \mid$ is introduced, the passage from $\tilde{X}_{i}$ to $X_{i}^{0}$ is manifest: 


$$
X_{i}^{0}=<\psi\left|\tilde{X}_{i}\right| \psi>=<\psi\left|L_{i}\right| \psi>=L_{i}+<\psi \mid\left[L_{i}, \mid \psi>\right]
$$

As a byproduct we have obtained a representation of the connection $A_{i}$ in terms of the vector-valued function $\mid \psi>$ :

$$
A_{i}=<\psi \mid\left[L_{i}, \mid \psi>\right]
$$

Before entering into details we firstly notice ( as in ref. [6] ) that the direct introduction of the link (3.8) between matrix model variables and projectors into the classical action (3.3) gives rise to a problem at a level of the variational principle. In fact the equations of motion obtained by varying the projector $p_{0}$, subject to the conditions $p_{0}^{2}=p_{0}, p_{0}^{\dagger}=p_{0}$, contain more solutions than those obtained by varying the connection $X_{i}^{0}$.

To avoid such ambiguity we will limit ourself to introduce the link with the projectors (3.8) at the level of Yang-Mills equations of motion and we will show that the monopole projectors are indeed solutions of them.

By varying the classical Yang-Mills action (3.3) with respect to $X_{i}^{0}$ we find the following equation:

$$
\left[X_{j}^{0}, F_{i j}\right]=i(\lambda-1) \epsilon_{i j k} F_{j k}
$$

where

$$
F_{i j}=\left[X_{i}^{0}, X_{j}^{0}\right]-i \epsilon_{i j k} X_{k}^{0}
$$

For $\lambda=2$ these matrix model equations of motion coincide precisely with those of pure Yang-Mills theory on a two-sphere, by simply posing the auxiliary scalar field $\phi=0$.

We are ready to introduce the constraint $\tilde{X}_{i}=p_{0} L_{i} p_{0}$ in (3.8) and to verify that the monopole projectors (2.17) and (2.22) are indeed solution of (3.12). Firstly we compute $F_{i j}$

$$
F_{i j}=p_{0}\left(\left[L_{i}, p_{0}\right]\left[L_{j}, p_{0}\right]-\left[L_{j}, p_{0}\right]\left[L_{i}, p_{0}\right]\right)
$$

where we made use of the property:

$$
p_{0}\left[L_{i}, p_{0}\right] p_{0}=0
$$


Therefore the equations (3.12), rewritten in terms of a generic projector $p_{0}$, take the following form:

$$
\begin{aligned}
& p_{0}\left[L^{j},\left(\left[L_{i}, p_{0}\right]\left[L_{j}, p_{0}\right]-\left[L_{j}, p_{0}\right]\left[L_{i}, p_{0}\right]\right)\right] p_{0}= \\
& =i(\lambda-1) \epsilon_{i j k} p_{0}\left(\left[L_{i}, p_{0}\right]\left[L_{j}, p_{0}\right]-\left[L_{j}, p_{0}\right]\left[L_{i}, p_{0}\right]\right) p_{0} .
\end{aligned}
$$

The evaluation of these equations on the classical projectors (2.17) and (2.22) is surprisingly more difficult than the noncommutative case as we shall see later. However we can verify directly (3.16) for the simplest case, the monopole with charge 1:

$$
p_{1}=\frac{1}{2}\left(\begin{array}{cc}
1+\cos \theta & \sin \theta e^{-i \phi} \\
\sin \theta e^{i \phi} & 1-\cos \theta
\end{array}\right) .
$$

After simple calculations we get

$$
\begin{aligned}
F_{\theta \phi} & =p_{1}\left(\partial_{\theta} p_{1} \partial_{\phi} p_{1}-\partial_{\phi} p_{1} \partial_{\theta} p_{1}\right)=\frac{i}{2} \sin \theta p_{1} \\
F_{i j} & =-\left(k_{i}^{\theta} k_{j}^{\phi}-k_{i}^{\phi} k_{j}^{\theta}\right) F_{\theta \phi}=-\frac{i}{2} p_{1} \epsilon_{i j k} \frac{x^{k}}{R} .
\end{aligned}
$$

It is then enough to apply the formula

$$
\left[L_{j}, x_{k}\right]=i \epsilon_{j k l} x^{l}
$$

and eq. (3.15) to verify that $p_{1}$ is indeed solution of the equations of motion (3.16) for $\lambda=2$.

Once clarified the interconnection between classical projectors and Y-M equations, it is interesting to reconstruct the monopole connections starting from the knowledge of the projectors $p_{ \pm n}$.

To reach this goal it is convenient to recall the formula (3.11)

$$
A_{i}=<\psi \mid\left[L_{i}, \mid \psi>\right]
$$

where the commutator action reduces to a derivative action in the classical case:

$$
\left[L_{i}, .\right]=-i\left(k_{i}^{\theta} \partial_{\theta}+k_{i}^{\phi} \partial_{\phi}+k_{i}^{\psi} \partial_{\psi}\right)
$$




$$
\begin{aligned}
k_{i}^{\theta} & =(-\sin \phi, \cos \phi, 0) \\
k_{i}^{\phi} & =(-\cos \phi \cot \theta \theta,-\sin \phi \cot g \theta, 1) \\
k_{i}^{\psi} & =\left(-\frac{\cos \phi}{\sin \theta},-\frac{\sin \phi}{\sin \theta}, 0\right)
\end{aligned}
$$

where $k_{i}^{\theta}, k_{i}^{\phi}$ are the killing vector of the sphere and $k_{i}^{\psi}$ is the residue of the extended action on $S^{3}$. Unfortunately the derivative action contains the dependence on the auxiliary $U(1)$ variable $\psi$ of the three sphere $S^{3}$, since the vector-valued function $<\psi$, by construction, depends on the total space $S^{3}$, while the projectors depend only on the physical base space $S^{2}$.

Therefore for the simplest monopole $n=1$

$$
<\psi_{1} \mid=\left(\bar{z}_{0}, \bar{z}_{1}\right)
$$

with

$$
z_{0}=\cos \frac{\theta}{2} e^{i\left(\frac{\psi-\phi}{2}\right)} \quad z_{1}=\sin \frac{\theta}{2} e^{i\left(\frac{\psi+\phi}{2}\right)}
$$

we obtain at first sight for the connection

$$
\begin{aligned}
& A_{i}=k_{i}^{\theta} A_{\theta}+k_{i}^{\phi} A_{\phi}+k_{i}^{\psi} A_{\psi} \\
& A_{\psi}=\frac{1}{2} \quad A_{\theta}=0 \quad A_{\phi}=-\frac{1}{2} \cos \theta
\end{aligned}
$$

We haven't yet been able to identify the monopole connection for the presence of the spurious component $A_{\psi}$. However since the field strength $F_{i j}$ depends only on the variables $\theta$ and $\phi$, being determined by the projectors $p_{ \pm n}$ as in the formula (3.14), it turns out that the presence of the component $A_{\psi}$ is purely fictitious and can be removed by redefining the vector-valued function $<\psi_{1} \mid$ :

$$
<\psi_{1}\left|\rightarrow<\psi_{1}^{\prime}\right|=e^{i \frac{\psi}{2}}<\psi_{1} \mid \quad \text { for } n=1
$$

or in the general case

$$
<\psi_{n}\left|\rightarrow<\psi_{n}^{\prime}\right|=e^{i n \frac{\psi}{2}}<\psi_{n} \mid \quad \forall n \in N .
$$

After this redefinition, which doesn't alter the projectors, we obtain the well-known result for the monopole connection: 


$$
A_{\theta}=0 \quad A_{\phi}=-\frac{n}{2} \cos \theta
$$

We have therefore learned that the Lie derivative action is equivalent to the ordinary derivate action $d=d \theta \frac{\partial}{\partial \theta}+d \phi \frac{\partial}{\partial \phi}$ if and only if the vector-valued function $<\psi_{n} \mid$ can be projected to the basic space $S^{2}$. This redefinition, which is simple in the classical case, turns out to be necessary also in the noncommutative case.

\section{Noncommutative projectors}

In Ref. 2] ( see also [3]-[5] ), we have been able to extend the classical monopoles in terms of new noncommutative projectors having as entries the elements of the fuzzy-sphere algebra:

$$
\begin{aligned}
& {\left[\hat{x}_{i}, \hat{x}_{j}\right]=i \alpha \epsilon_{i j k} \hat{x}_{k} \quad \sum_{i}\left(\hat{x}_{i}\right)^{2}=R^{2}} \\
& \alpha=\frac{2 R}{\sqrt{N(N+2)}}
\end{aligned}
$$

In the $N \rightarrow \infty$ limit this algebra reduces to the classical two-sphere. Our construction is based on the observation that the classical $x_{i}$ coordinates are related to a couple of complex coordinates by the Hopf principal fibration. The noncommutativity between the coordinates is then realized by promoting the two complex coordinates to a couple of independent oscillators:

$$
\begin{aligned}
z_{i} & \rightarrow a_{i} \quad\left[a_{i}, a_{j}^{\dagger}\right]=\delta_{i j} \\
\hat{x}_{1} & =\frac{\hat{\alpha}}{2}\left(a_{0} a_{1}^{\dagger}+a_{1} a_{0}^{\dagger}\right) \\
\hat{x}_{2} & =i \frac{\hat{\alpha}}{2}\left(a_{0} a_{1}^{\dagger}-a_{1} a_{0}^{\dagger}\right) \\
\hat{x}_{3} & =\frac{\hat{\alpha}}{2}\left(a_{0} a_{0}^{\dagger}-a_{1} a_{1}^{\dagger}\right) \\
\hat{N} & =a_{0}^{\dagger} a_{0}+a_{1}^{\dagger} a_{1} .
\end{aligned}
$$

The operator $\hat{\alpha}$ is equal to $\alpha$ for representations with fixed total oscillator number $\hat{N}=N$, which is the characteristic noncommutative parameter of the fuzzy sphere. As it happens in the principal Hopf fibration we can redefine the oscillators with a $U(1)$ factor which is cancelled in the combinations $\hat{x}_{i}$. 
Basically, the projectors are constructed in terms of vector-valued operators $<\psi \mid$, taking values in the oscillator algebra, while the projectors will be dependent on polynomial functions of the fuzzy-sphere algebra $\hat{x}_{i}$ only.

To construct the projectors $p_{n}\left(\hat{x}_{i}\right)$ we have considered the $(n+1)$-dimensional vectors:

$$
\mid \psi_{n}>=N_{n}\left(\begin{array}{c}
\left(a_{0}\right)^{n} \\
\ldots . \\
\sqrt{\left(\begin{array}{c}
n \\
k
\end{array}\right)}\left(a_{0}\right)^{n-k}\left(a_{1}\right)^{k} \\
\ldots \ldots \\
\left(a_{1}\right)^{n}
\end{array}\right) .
$$

Constraining the vector $<\psi_{n} \mid$ to be normalized one notice that the function $N_{n}$ is fixed to be dependent only on the number operator $\hat{N}$

$$
\begin{aligned}
& <\psi_{n} \mid \psi_{n}>=1 \\
& N_{n}=N_{n}(\hat{N})=\frac{1}{\sqrt{\prod_{i=0}^{n-1}(\hat{N}-i+n)}}
\end{aligned}
$$

Then the $n$-monopoles projector is simply

$$
p_{n}=\left|\psi_{n}><\psi_{n}\right|
$$

and it satisfies the basic properties of a projector, due to the normalization condition (4.4).

It is easy to notice that in the ket-bra product there appear only combinations of oscillators, commuting with the number operator $\hat{N}$, and therefore the action of $p_{n}$ can be restricted to a fixed value $\hat{N}=N$ and its entries belong to the fuzzy-sphere algebra.

Moreover the projector $P_{n}$ has a positive trace given by

$$
\operatorname{Tr} p_{n}=\operatorname{Tr}\left|\psi_{n}><\psi_{n}\right|=\frac{N+n+1}{N+1} \operatorname{Tr} I=N+n+1<\operatorname{Tr} I=(N+1)(n+1) .
$$

Being the algebra of the fuzzy sphere a finite-dimensional algebra, the trace of the projector $p_{n}$ is always a positive integer and less than the trace of the identity. 
To construct the solution for $p_{-n}(n>0)$ it is enough to take the adjoint of the components of the vector (4.3), apart the normalization factor, which is different in this case. Consider the $(n+1)$-dimensional vectors:

$$
\mid \psi_{-n}>=N_{n}\left(\begin{array}{c}
\left(a_{0}^{\dagger}\right)^{n} \\
\ldots \ldots \\
\sqrt{\left(\begin{array}{c}
n \\
k
\end{array}\right)}\left(a_{0}^{\dagger}\right)^{n-k}\left(a_{1}^{\dagger}\right)^{k} \\
\ldots \ldots \\
\left(a_{1}^{\dagger}\right)^{n}
\end{array}\right) .
$$

Again normalizing the vector $<\psi_{-n} \mid$ fixes the function $N_{n}$ to be dependent only on the number operator:

$$
\begin{aligned}
& <\psi_{-n} \mid \psi_{-n}>=1 \\
& N_{n}=N_{n}(\hat{N})=\frac{1}{\sqrt{\prod_{i=0}^{n-1}(\hat{N}+i+2-n)}} .
\end{aligned}
$$

The corresponding projector $p_{-n}$ doesn't exist for all values of $n$, but only for $0<n<$ $N+2$. In fact the corresponding trace is given by:

$$
\operatorname{Tr} p_{-n}=\operatorname{Tr}\left|\psi_{-n}><\psi_{-n}\right|=\frac{N+1-n}{N+1} \operatorname{Tr} I=N+1-n<\operatorname{Tr} I .
$$

This trace is an integer, but it is positive definite if and only if the following bound is respected:

$$
n<N+1
$$

For the special case $n=N+1$ we simply obtain a null projector.

In summary the projectors $p_{ \pm n}\left(\hat{x}_{i}\right)$ have the nice property to be a smooth deformation of the classical projectors $p_{ \pm n}\left(x_{i}\right)$, making evident the existence of noncommutative monopoles which tends for $N \rightarrow \infty$ to the classical ones. We recall that this procedure has been successfully extended to the fuzzy four-sphere case [22].

To construct the corresponding connection we need more work because we must be sure that the action of the Lie derivative $\left[L_{i},.\right]$ on the vectors $\left|\psi_{ \pm n}\right\rangle$ has as a smooth limit the 
classical Lie derivative on the sphere and this criterium requires an adjustment of the present construction.

\section{Properties of noncommutative projectors}

Let us start from the generic noncommutative projectors $p_{n}$; defining the corresponding matrix model variables as in eq. (3.8), we easily obtain, using the oscillator algebra, *

$$
\begin{aligned}
\tilde{X}_{i}^{(n)}= & p_{n} L_{i} p_{n}=\frac{N}{N+n}\left|\psi_{n}>L_{i}<\psi_{n}\right|=\left|\psi_{n}>L_{i}<\psi_{n}\right|+\left|\psi_{n}>A_{i}^{(n)}<\psi_{n}\right| n>0 \\
\tilde{X}_{i}^{(-n)}= & p_{(-n)} L_{i} p_{(-n)}=\frac{N+2}{N+2-n}\left|\psi_{(-n)}>L_{i}<\psi_{(-n)}\right|=\left|\psi_{(-n)}>L_{i}<\psi_{(-n)}\right|+ \\
& +\left|\psi_{(-n)}>A_{i}^{(-n)}<\psi_{(-n)}\right| \quad 0<n<N+1 .
\end{aligned}
$$

The (gauge invariant ) connection is not only proportional to the classical monopole charge $n$ but it has another dependence from $n$ in the denominator:

$$
\begin{aligned}
\left|\psi_{n}>A_{i}^{(n)}<\psi_{n}\right|= & -\frac{n}{N+n}\left|\psi_{n}>L_{i}<\psi_{n}\right| n>0 \\
\left|\psi_{(-n)}>A_{i}^{(-n)}<\psi_{(-n)}\right|= & \frac{n}{N+2-n}\left|\psi_{(-n)}>L_{i}<\psi_{(-n)}\right| \\
& 0<n<N+1 .
\end{aligned}
$$

Let's see what happens for the field strength:

$$
\begin{aligned}
\tilde{F}_{i j}^{(n)}= & -\frac{n N}{(N+n)^{2}} i \epsilon_{i j k}\left|\psi_{n}>L_{k}<\psi_{n}\right| \quad n>0 \\
\tilde{F}_{i j}^{(-n)}= & \frac{n(N+2)}{(N+2-n)^{2}} i \epsilon_{i j k}\left|\psi_{(-n)}>L_{k}<\psi_{(-n)}\right| \\
& 0<n<N+1 .
\end{aligned}
$$

Differently from the classical case, the field strength is no more simply proportional to the instanton number $n$ unless in the $N \rightarrow \infty$ limit; this is the main obstacle to define

*For the simplest case $n=1, N=1$ we have checked that the result can be expressed as a sum of two independent angular momenta, one is intrinsic to the fuzzy sphere algebra $L_{i} \otimes 1$ and the other $1 \otimes S_{i}$ acting on the $(n+1) \otimes(n+1)$ auxiliary representation space of the projector. 
an integer Chern class for the noncommutative monopole, due to the nonlinear additional contribution.

The corresponding equations of motion are solved by a certain value of $\lambda$, due to the identity:

$$
\begin{aligned}
& {\left[\tilde{X}_{j}^{(n)}, \tilde{F}_{i j}^{(n)}\right]=i \frac{N}{N+n} \epsilon_{i j k} \tilde{F}_{j k}^{(n)} \quad \lambda=2-\frac{n}{N+n} \quad n>0} \\
& {\left[\tilde{X}_{j}^{(-n)}, \tilde{F}_{i j}^{(-n)}\right]=i \frac{N+2}{N+2-n} \epsilon_{i j k} \tilde{F}_{j k}^{(-n)} \quad \lambda=2+\frac{n}{N+2-n} \quad 0<n<N+1}
\end{aligned}
$$

We see therefore that the class of models we are interested in is situated around the classical value $\lambda=2$ confirming an interpretation already outlined in our paper 9]. In particular we noticed that to define noncommutative soliton solutions, having a smooth limit to the classical ones, it was necessary to perturb the $\lambda$ coupling constant in a similar form:

$$
\lambda=\lambda_{c l}+\frac{c}{N}
$$

For the special case $\lambda_{c l}=2$ we already found a class of solutions tending to the classical solution $\phi=$ const., $A_{a}=0$; in this case the matrix model variable $X_{i}$ was equal to a rescaling of the background solution by a factor $\left(1+f\left(\frac{1}{N}\right)\right)$ :

$$
X_{i}=\left(1+f\left(\frac{1}{N}\right)\right) \hat{x}_{i} \quad \rightarrow \quad \phi=\text { const } \quad A_{a}=0 .
$$

Evidently, inside the class of models (5.4) it is possible to reach a different classical limit:

$$
X_{i} \rightarrow \phi=0, \quad A_{a}=\text { monopole }
$$

and we will discuss later how this fact happens. We have already reached an important result, i.e. we know for what class of models our noncommutative projectors are solutions of the YM equations of motion. 


\section{Reconstruction of the gauge connection from projec- tors}

The reconstruction of the gauge connection from the noncommutative projectors requires another step, since the vector $<\psi_{n} \mid$, used for the computation in (4.3), is function of the oscillator algebra, which is more general of the fuzzy sphere algebra. This produces a discontinuity problem in the classical limit. In fact the Lie derivative, when it acts on the oscillator algebra, has the form

$$
\begin{aligned}
L_{x} & =\frac{1}{2}\left[a_{0} a_{1}^{\dagger}+a_{1} a_{0}^{\dagger}, .\right] \\
L_{y} & =\frac{1}{2}\left[i\left(a_{0} a_{1}^{\dagger}-a_{1} a_{0}^{\dagger}\right), .\right] \\
L_{z} & =\frac{1}{2}\left[a_{0} a_{0}^{\dagger}-a_{1} a_{1}^{\dagger}, .\right]
\end{aligned}
$$

and it can be represented in the classical limit with the extended action:

$$
\begin{aligned}
L_{i} & =-i\left(k_{i}^{\theta} \partial_{\theta}+k_{i}^{\phi} \partial_{\phi}+k_{i}^{\psi} \partial_{\psi}\right) \\
k_{i}^{\theta} & =(-\sin \phi, \cos \phi, 0) \\
k_{i}^{\phi} & =(-\cos \phi \operatorname{cotg} \theta,-\sin \phi \operatorname{cotg} \theta, 1) \\
k_{i}^{\psi} & =\left(-\frac{\cos \phi}{\sin \theta},-\frac{\sin \phi}{\sin \theta}, 0\right) .
\end{aligned}
$$

As a consequence, a spurious dependence on the variable $\psi \in S^{3}$ is generated, which is physically irrelevant since the field strength is function only of the two-sphere $S^{2}$.

At the classical level we already discussed such problem and we have observed that the vector $|\psi\rangle$ has a sort of $U(1)$ gauge arbitrariness $|\psi\rangle \rightarrow \mid \psi>e^{i \phi}$ leaving the projector invariant.

What is the analogue of this phase ambiguity at a noncommutative level ? Let's write for example the vector-valued operator $\mid \psi>$ in the case $n=-1$ :

$$
\begin{aligned}
\mid \psi_{n=-1}> & =\frac{1}{\sqrt{\hat{N}+1}}\left(\begin{array}{c}
\bar{a}_{0} \\
\bar{a}_{1}
\end{array}\right)=\left(\begin{array}{c}
\tilde{\bar{a}}_{0} \\
\tilde{\bar{a}}_{1}
\end{array}\right) \\
\tilde{\bar{a}}_{0} & =\sum_{n_{1}=0}^{\infty} \sum_{n_{2}=0}^{\infty} \sqrt{\frac{n_{1}+1}{n_{1}+n_{2}+2}}\left|n_{1}+1, n_{2}><n_{1}, n_{2}\right|
\end{aligned}
$$




$$
\begin{aligned}
\tilde{\bar{a}}_{1} & =\sum_{n_{1}=0}^{\infty} \sum_{n_{2}=0}^{\infty} \sqrt{\frac{n_{2}+1}{n_{1}+n_{2}+2}}\left|n_{1}, n_{2}+1><n_{1}, n_{2}\right| \\
<\psi_{n=-1} \mid & =\left(a_{0}, a_{1}\right) \frac{1}{\sqrt{\hat{N}+1}}=\left(\tilde{a}_{0}, \tilde{a}_{1}\right) \\
\tilde{a}_{0} & =\sum_{n_{1}=0}^{\infty} \sum_{n_{2}=0}^{\infty} \sqrt{\frac{n_{1}+1}{n_{1}+n_{2}+2}}\left|n_{1}, n_{2}><n_{1}+1, n_{2}\right| \\
\tilde{a}_{1} & =\sum_{n_{1}=0}^{\infty} \sum_{n_{2}=0}^{\infty} \sqrt{\frac{n_{2}+1}{n_{1}+n_{2}+2}}\left|n_{1}, n_{2}><n_{1}, n_{2}+1\right| \\
<\psi_{-1} \mid \psi_{-1}> & =1 .
\end{aligned}
$$

The problem which complicates the classical limit is that the action of $\left|\psi_{-1}\right\rangle$ doesn't commute with the number operator $\hat{N}$ and therefore it is not possible to restrict its action to a fixed number $N$, as instead we have done for the projectors. It is then necessary to correct the vector $\left|\psi_{-1}\right\rangle$ with an operator, acting on the right and not commuting with the number operator, i.e. a quasi-unitary operator:

$$
\left|\psi_{-1}>\rightarrow\right| \psi_{-1}^{\prime}>=\mid \psi_{-1}>U \quad U U^{\dagger}=1 \quad\left(U^{\dagger} U=1-P_{0}\right)
$$

in order to keep invariant the noncommutative projectors. The presence of the quasiunitary operator $U$ adjusts the classical limit, making possible to extrapolate the noncommutative connection. Many choices for $U$ are possible, for example:

$$
U_{1}=\sum_{n_{1}=0}^{\infty} \sum_{n_{2}=0}^{\infty}\left|n_{1}, n_{2}><n_{1}+1, n_{2}\right|
$$

or

$$
U_{2}=\sum_{n_{1}=0}^{\infty} \sum_{n_{2}=0}^{\infty}\left|n_{1}, n_{2}><n_{1}, n_{2}+1\right|
$$

are equally good, since the difference between these two operators is a gauge transformation of the connection. Let's compute

$$
\begin{aligned}
\mid \psi_{-1}^{\prime}> & =\mid \psi_{-1}>U_{1}=\left(\begin{array}{c}
\tilde{\overline{a^{\prime}}} \\
\tilde{\overline{a^{\prime}}}
\end{array}\right) \\
{\tilde{\overline{a^{\prime}}}}_{0} & =\sum_{n_{1}=0}^{\infty} \sum_{n_{2}=0}^{\infty} \sqrt{\frac{n_{1}+1}{n_{1}+n_{2}+2}}\left|n_{1}+1, n_{2}><n_{1}+1, n_{2}\right|
\end{aligned}
$$




$$
{\tilde{\overline{a^{\prime}}}}_{1}=\sum_{n_{1}=0}^{\infty} \sum_{n_{2}=0}^{\infty} \sqrt{\frac{n_{2}+1}{n_{1}+n_{2}+2}}\left|n_{1}, n_{2}+1><n_{1}+1, n_{2}\right| \text {. }
$$

At this point $\left[\hat{N},\left|\psi_{-1}^{\prime}\right\rangle\right]=0$ and we can truncate the action of $\left|\psi_{-1}^{\prime}\right\rangle$ to a fixed number $N$ :

$$
\begin{aligned}
& \left.\tilde{\overline{a^{\prime}}}\right|_{N}=\sum_{k=0}^{N-1} \sqrt{\frac{k+1}{N+1}}|k+1, N-k-1><k+1, N-k-1| \\
& \left.\tilde{\bar{a}^{\prime}}\right|_{N}=\sum_{k=0}^{N-1} \sqrt{\frac{N-k}{N+1}}|k, N-k><k+1, N-k-1| .
\end{aligned}
$$

These actions can be reexpressed in terms of spherical harmonics of the fuzzy sphere, i.e. the physical functional space of the noncommutative case.

We can verify that $\left|\psi_{-1}^{\prime}\right\rangle$ gives rise to connections satisfying the Y-M equations of motion ( in the gauge-covariant formulation ). Since

$$
\tilde{X}_{i}=\left|\psi_{-1}>U \frac{N+2}{N+1}\left(U^{\dagger} L_{i} U\right) U^{\dagger}<\psi_{-1}\right|=\left|\psi_{-1}^{\prime}>X_{i}<\psi_{-1}\right|
$$

we can deduce that the physical matrix model variable $X_{i}$ is of the form:

$$
\begin{aligned}
& X_{i}=\frac{N+2}{N+1} U^{\dagger} L_{i} U \\
& F_{i j}=\left[X_{i}, X_{j}\right]-i \epsilon_{i j k} X_{k}=\frac{N+2}{(N+1)^{2}} i \epsilon_{i j k}\left(U^{\dagger} L_{j} U\right) \\
& {\left[X^{j}, F_{i j}\right]=i(\lambda-1) \epsilon_{i j k} F_{j k} \quad \lambda=2+\frac{1}{N+1} .}
\end{aligned}
$$

The generalization of these results to the case $\left|\psi_{-n}\right\rangle$ with $n$ generic $(n<N+1)$ is straightforward.

We can summarize these results as follows. The matrix model solution $X_{i}$ for charge $-n$ is obtained in two steps:

i) re-scaling the background solution $X_{i}^{(0)}=\frac{N+2}{N+2-n} L_{i}$, as already noticed in the appendix of the paper [9], leading to the classical solution $\phi=$ const., $A_{a}=0$;

ii) dressing with the quasi-unitary operator $X_{i}=U^{\dagger} X_{i}^{(0)} U$, shifting the solution in another class with respect to $i$ ), the monopole class. Therefore the noncommutative map between the two classes is realized with a quasi-unitary operator 


$$
U:\left(\phi=\text { const., } A_{a}=0\right) \rightarrow\left(\phi=0, A_{a}=\text { monopole }\right)
$$

We note that the action of quasi-unitary operators on the background generates reducible representations of the $S U(2)$ Lie algebra, revealing their topological character. Therefore the classification of nontrivial topologies on a fuzzy sphere is reduced to the classification of reducible representations of the $S U(2)$ Lie algebra ${ }^{\dagger}$.

It remains to be investigated if this construction can be repeated for the case $\left.\left|\psi_{n}\right\rangle, n\right\rangle$ 0 , for example $n=1$ :

$$
\begin{aligned}
& \mid \psi_{n=1}>=\frac{1}{\sqrt{\hat{N}+1}}\left(\begin{array}{c}
a_{0} \\
a_{1}
\end{array}\right)=\left(\begin{array}{c}
\tilde{a}_{0} \\
\tilde{a}_{1}
\end{array}\right) \\
& \tilde{a}_{0}=\sum_{n_{1}=0}^{\infty} \sum_{n_{2}=0}^{\infty} \sqrt{\frac{n_{1}+1}{n_{1}+n_{2}+1}}\left|n_{1}, n_{2}><n_{1}+1, n_{2}\right| \\
& \tilde{a}_{1}=\sum_{n_{1}=0}^{\infty} \sum_{n_{2}=0}^{\infty} \sqrt{\frac{n_{2}+1}{n_{1}+n_{2}+1}}\left|n_{1}, n_{2}><n_{1}, n_{2}+1\right| \\
& <\psi_{n=1} \mid=\frac{1}{\sqrt{\hat{N}+1}}\left(\bar{a}_{0}, \bar{a}_{1}\right)=\left(\tilde{\bar{a}}_{0}, \tilde{\bar{a}}_{1}\right) \\
& \tilde{\bar{a}}_{0}=\sum_{n_{1}=0}^{\infty} \sum_{n_{2}=0}^{\infty} \sqrt{\frac{n_{1}+1}{n_{1}+n_{2}+1}}\left|n_{1}+1, n_{2}><n_{1}, n_{2}\right| \\
& \tilde{\bar{a}}_{1}=\sum_{n_{1}=0}^{\infty} \sum_{n_{2}=0}^{\infty} \sqrt{\frac{n_{2}+1}{n_{1}+n_{2}+1}}\left|n_{1}, n_{2}+1><n_{1}, n_{2}\right| \\
& <\psi_{1}\left|\psi_{1}>=1-\right| 0,0><0,0 \mid=1-P_{0} \text {. }
\end{aligned}
$$

The last normalization condition is equivalent to the identity since:

$$
\left|\psi_{1}>P_{0}=P_{0}<\psi_{1}\right|=0
$$

the action of $\mid \psi_{1}>$ on the projector $P_{0}$ is null.

If we try to redefine $\left|\psi_{1}\right\rangle$ in order to commute with the number operator, we are forced to introduce the adjoint of the quasi-unitary operator $U$

$$
U^{\dagger}=\sum_{n_{1}=0}^{\infty} \sum_{n_{2}=0}^{\infty}\left|n_{1}+1, n_{2}><n_{1}, n_{2}\right| .
$$

${ }^{\dagger}$ Since there are no one-dimensional representations of $S U(2)$ Lie algebra, the case $n=N$ leads to a vanishing matrix variable, also if the corresponding projector is non-vanishing. 
However in this case the dressing unfortunately alters the form of the projector $p_{1}$ since

$$
\left|\psi_{1}^{\prime}><\psi_{1}^{\prime}\right|=\left|\psi_{1}>U^{\dagger} U<\psi_{1}\right|=\left|\psi_{1}>\left(1-\sum_{n=0}^{\infty}|0, n><0, n|\right)<\psi_{1}\right| .
$$

In fact the extra contribution is not cancelled by the presence of $\left|\psi_{1}\right\rangle$. We have checked, for the special case $N=1$ using the basis of Pauli matrices, that only the combination $1-p_{1}$ can be written as a ket-bra valued operator, but unfortunately $1-p_{1}$ doesn't satisfy the Y-M equations of motion. We therefore find a contradictory result, since it is impossible for the charge $n$ monopoles to define a connection satisfying the Y-M equations of motion, while the corresponding projector does it. We conclude that at noncommutative level there is in general no equivalence relation between projectors and connections, as it happens in the classical case.

\section{Conclusions}

This work clarifies how to introduce topologically nontrivial configurations on the fuzzy sphere. In particular it reveals the mechanism with which the noncommutative topology can smoothly extend the commutative one. In Ref. 2] we were able to construct some noncommutative projectors having as entries the elements of the fuzzy sphere algebra, and tending smoothly in the $N \rightarrow \infty$ limit to the classical monopoles on the sphere $S^{2}$. However at a physical level the classical monopoles are also solutions of the equations of motion of the $Y-M$ action on the sphere. Our first result is to identify a class of models for which the noncommutative projectors [2] are solutions of the corresponding equations of motion.

In the spirit of the Serre-Swan theorem we have then tried to reconstruct the gauge connection or equivalently the matrix model variables $X_{i}$ corresponding to these projectors. In this sense we have reached a partially successful result because it is possible to deconstruct only the projectors with negative charge $-k\left(p_{-k}, k>0\right)$, but not those of positive charge $p_{k}, k>0$.

In the $p_{-k}(k>0)$ case, we have isolated the corresponding matrix model solution, which results to be composed in two steps:

i) with a re-scaling of the background solution, which is necessary to require that the noncommutative topology has as a classical smooth limit the commutative one;

ii) dressing with a quasi-unitary operator, that maps the background, which is an irreducible representation of the $S U(2)$ Lie algebra, to a reducible representation. Therefore, at 
a level of the matrix model, the classification of the topologically nontrivial configurations is reduced to the classification of the reducible representations of the $S U(2)$ Lie algebra, at least in the fuzzy sphere case. The fact that we are able to find connections only for negative charge projectors $p_{-k}(0<k<N+1)$ is explained with the fact that in a $(N+1) \times(N+1)$ matrix variable $X_{i}$ we can insert a ( reducible ) representation with rank less than $(N+1)$, but not bigger than $(N+1)$.

The case of positive charge projectors $p_{k}(k>0)$, where it is not possible to define a corresponding connection satisfying the $Y-M$ equations of motion is a counterexample to an eventual equivalence relation between projectors and connections, as instead it happens in the classical case.

\section{References}

[1] J. Madore, " The Fuzzy sphere ", Class. Quantum Grav. 9 (1992) 69.

[2] P. Valtancoli, " Projectors for the fuzzy sphere " Mod.Phys.Lett. A16 (2001) 639, hep-th/0101189.

[3] S. Baez, A.P. Balachandran, B. Ydri, S. Vaidya," Monopoles and Solitons in fuzzy physics ", Comm. Math. Phys. 208 (2000) 787, hep-th/9811169.

[4] A.P. Balachandran and S. Vaidya, " Instantons and chiral anomaly in fuzzy physics ", hep-th/9910129.

[5] A. P. Balachandran, X.Martin and D. O' Connor, " Fuzzy actions and their continuum limits ", hep-th/0007030.

[6] M.Dubois-Violette, Y. Georgelin, " Gauge theory in terms of projector valued fields ", Phys. Lett. B 82 251, 1979.

[7] T. Eguchi, P. B. Gilkey, A. J. Hanson, " Gravitation, gauge theories and differential geometry ", Phys.Rept. 66 213,1980.

[8] P. Valtancoli, " Stability of the fuzzy sphere solution from matrix model", Int. J. Mod. Phys. A18 (2003), 967; hep-th/0206075

[9] P. Valtancoli, " Solitons for the fuzzy sphere from matrix model", Int. J. Mod. Phys. A18 (2003), 1107; hep-th/0209117.

[10] S. Iso, Y. Kimura, K. Tanaka and K. Wakatsuki, " Noncommutative gauge theory on fuzzy sphere from Matrix model ", Nucl. Phys. B604 (2001) 121, hep-th/0101102. 
[11] R.C. Myers, "Dielectric branes", JHEP 9912 (1999) 022, hep-th/9910053.

[12] Y. Kimura, " Noncommutative gauge theories on fuzzy sphere and fuzzy torus from Matrix model ", Prog. Theor. Phys. 106 (2001) 445, hep-th/0103192.

[13] D. Berenstein, J. M. Maldacena, H. Nastase, " Strings in flat space and PP waves from N=4 Superyang-mills " , JHEP 0204 (2002) 013, hep-th/0202021.

[14] J. Ambjorn, Y.M. Makeenko, J. Nishimura and R. J. Szabo, " Finite N Matrix models of noncommutative gauge theory", JHEP 9911 (1999) 029, hep-th/9911041.

[15] J. Ambjorn, Y.M. Makeenko, J. Nishimura and R. J. Szabo, " Non perturbative Dynamics of noncommutative gauge theory", Phys. Lett. B480 (2000) 399.

[16] J. Ambjorn, Y.M. Makeenko, J. Nishimura and R. J. Szabo, " Lattice gauge fields and discrete noncommutative Yang-Mills Theory ", JHEP 0005 (2000) 023, hep-th/0004147.

[17] H. Aoki, S. Iso, H, Hawai, Y. Kitazawa, T. Tada and A. Tsuchiya, " IIB matrix model ", Prog. Theor. Phys. Suppl. 134 (1999) 47, hep-th/9908038.

[18] H. Aoki, S. Iso, H. Hawai, Y. Kitazawa and T. Tada," Space-time structures from IIB Matrix model ", Prog. Theor. Phys. 99 ( 1998) 713, hep-th/9802085.

[19] M. Li, " Strings from IIB matrices ", Nucl. Phys. B499(1997) 149, hep-th/9612222

[20] H. Aoki, N. Ishibashi, S. Iso, H. Kawai, Y. Kitazawa and T. Tada, " Noncommutative Tang-Mills in IIB Matrix model ", Nucl. Phys. B565 (2000) 176, hep-th/9908141.

[21] G. Landi, " Projective modules of finite type and monopoles over $S^{2}$ ", J.Geom.Phys. 37 47, 2001, math-ph/9905014.

[22] P. Valtancoli, " Projective modules over the fuzzy four sphere", Mod. Phys. Lett. A17 (2002) 2189; hep-th/0210166. 